\title{
RNAi-mediated A disintegrin and metalloproteinase 9 gene silencing inhibits the tumor growth of non-small lung cancer in vitro and in vivo
}

\author{
LIANG CHANG, FANGCHAO GONG and YOUBIN CUI \\ Department of Thoracic Surgery, The First Hospital, Jilin University, Changchun, Jilin 130021, P.R. China
}

Received May 28, 2014; Accepted February 19, 2015

DOI: $10.3892 / \mathrm{mmr} .2015 .3477$

\begin{abstract}
A disintegrin and metalloproteinase 9 (ADAM9) is a type I transmembrane protein that has been associated with cancer development and metastasis in various types of cancer. However, little is known about its role in non-small cell lung cancer (NSCLC). The aim of the present study was to evaluate whether downregulation of ADAM9 affects cell proliferation, apoptosis, migration and invasion in NSCLC. Thus, a recombinant lentiviral small hairpin RNA expression vector carrying ADAM9 was constructed and infected into the human NSCLC cell line A549. Cell proliferation, apoptosis, migration and invasion in vitro and tumor growth in vivo were determined following downregulation of ADAM9 by RNA interference-mediated ADAM9 gene silencing. It was found that downregulation of ADAM9 expression using an RNA silencing approach in A549 tumor cells significantly inhibited cell proliferation, migration and invasion, induced cell apoptosis in vitro, as well as suppressed in vivo tumor growth in an experimental mouse model of lung metastasis. These data indicate that ADAM9 is potentially an important new therapeutic target for the prevention of tumor growth in NSCLC.
\end{abstract}

\section{Introduction}

Lung cancer remains the leading cause of cancer-associated mortality in China and numerous other countries in the world (1). Non-small cell lung cancer (NSCLC) accounts for $\sim 85 \%$ of all types of lung cancer (2), with $<15 \%$ of patients surviving beyond 5 years $(3,4)$. Metastasis is a major cause of morbidity and mortality in NSCLC cancer. Surgical resection of primary lung cancer is frequently followed by tumor recurrence at distant sites, including the lymph nodes (5), bone (6) and brain (7). Therefore, in depth investigations into

Correspondence to: Professor Youbin Cui, Department of Thoracic Surgery, The First Hospital, Jilin University, 71 Xinmin Street, Chaoyang, Changchun, Jilin 130021, P.R. China

E-mail: cuiyoubin14528@sina.com

Key words: tumor growth, non-small lung cancer, A disintegrin and metalloproteinase 9, RNA silencing the mechanisms underlying NSCLC invasion and metastasis are required to gain insights into disease progression and to identify potential therapeutic targets and treatment strategies.

A disintegrin and metalloproteinases (ADAMs) comprise a family of type I transmembrane proteins containing a metalloproteinase and disintegrin extracellular domain, and are involved in cell adhesion, migration, cell signal transduction and the proteolytic processing of multiple transmembrane proteins (8). In addition, ADAMs are important in a range of human diseases, including inflammatory diseases and asthma $(9,10)$. ADAM family proteins, including ADAM9, ADAM12, ADAM15 and ADAM17 have also been associated with human cancer metastasis, formation and progression $(11,12)$.

Among these members of the ADAM protein family, ADAM9 in particular has been associated with tumorigenesis and tumor cell metastasis (13). It has been demonstrated that ADAM9 expression is frequently upregulated in various types of cancer, including breast cancer (14), hepatocellular carcinoma (15), gastric cancer (16), pancreatic ductal adenocarcinoma (17), prostate cancer (18), renal cell carcinoma (19) and cervical squamous carcinoma (20), correlating with cancer progression, metastasis and predicting a shortened survival time in patients (14-20). Consistent with these results, a previous study demonstrated that ADAM9 is highly expressed in NSCLC and highly expressed ADAM9 correlates with a shortened survival time (21). Notably, Shintani et al found that ADAM9 overexpression enhances cell adhesion and invasion of NSCLC cells via modulation of other adhesion molecules and altering sensitivity to growth factors (22). A previous study demonstrated that RNA interference (RNAi)-mediated downregulation of endogenous $A D A M 9$ could inhibit adenoid cystic carcinoma cell growth and metastasis in vitro and in vivo (23). The generation of MDA-MB-231 knockdown clones lacking ADAM9 expression inhibited breast cancer cell invasion in vitro (24). The above studies imply that ADAM9 is important in tumor metastasis.

However, relatively little is known about the role of ADAM9 in NSCLC cells. Thus, in the present study, the feasibility of lentiviral vector delivered small hairpin RNA (shRNA) against ADAM9 for the treatment of NSCLC in vitro and in vivo was assessed and the molecular pathways involved were analyzed. 


\section{Materials and methods}

Materials. Dulbecco's modified Eagle's medium (DMEM) and fetal bovine serum (FBS) were purchased from HyClone (Logan, UT, USA).Lipofectamine ${ }^{\mathrm{TM}} 2000$ and TRIzol ${ }^{\circledR}$ reagent $^{2}$ was obtained from Invitrogen Life Technologies (Carlsbad, CA, USA). Moloney murine leukemia virus (M-MLV) reverse transcriptase was purchased from Promega (Madison, WI, USA). All other chemicals were obtained from Sigma-Aldrich (St. Louis, MO, USA). For western blot analysis, the following primary antibodies were used: Mouse monoclonal anti-human $\beta$-actin (1:5,000 dilution; cat. no. A2228; Sigma-Aldrich) and mouse monoclonal anti-human ADAM9 $(1 ; 2,000$ dilution; cat. no. sc-23290) purchased from Santa Cruz Biotechnology, Inc. (Santa Cruz, CA, USA).

Cell culture. The human non-small-cell lung cancer cell lines A549 and human embryonic kidney 293T cell lines were purchased from the Cell Bank of Type Culture Collection of the Chinese Academy of Sciences, Shanghai Institute of Cell Biology, Chinese Academy of Sciences (Shanghai, China). A549 cells and 293T cells were cultured in DMEM supplemented with heat-inactivated $10 \% \mathrm{FBS}$ at $37^{\circ} \mathrm{C}$ in a humidified atmosphere containing $5 \% \mathrm{CO}_{2}$.

Construction of the ADAM9 shRNA lentivirus vector and cell infection. The following oligonucleotides were synthesized. The sequence for the negative control small interfering RNA (siRNA) was 5'-AATTCTCCGAACGTGTCACGT-3', which did not target any genes in humans, mice or rats and was essential for determining the effects of siRNA delivery. The ADAM9 siRNA sequence was 5'-GGCGGGATTAATGTGTTTG-3'. The stem-loop-stem oligos (shRNAs) were synthesized, annealed and ligated into the NheI/Pac I-linearized pFH-Lvector. The lentiviral-based shRNA-expressing vectors were confirmed by DNA sequencing. The generated plasmids were referred to as $\mathrm{pFH}$-LshADAM9 or $\mathrm{pFH}$-LshCon, respectively. Recombinant lentiviral vectors and packaging vectors were then transfected into 293T cells via Lipofectamine ${ }^{\mathrm{TM}} 2000$ (Invitrogen Life Technologies) to generate the lentivirus. Supernatants containing either the lentivirus expressing the ADAM9 shRNA or the control shRNA were harvested $72 \mathrm{~h}$ after transfection. The lentiviruses were purified by ultracentrifugation at $17,000 \mathrm{xg}$ for $30 \mathrm{~min}$ and the titer of the lentiviruses was determined.

For lentivirus infection, A549 cells were cultured in 6-well plates. Subsequently, ADAM9 shRNA-expressing lentivirus or non-targeting shRNA-expressing lentivirus (negative control) was added, with a multiplicity of infection of 10 in A549 cells, and mock-infected cells were used as negative controls. After 3 days of infection, cells were observed under a fluorescence microscope (MicroPublisher 3.3 RTV; Olympus, Tokyo, Japan).

Reverse transcription quantitative polymerase chain reaction $(R T-q P C R)$ analysis. Total RNA was extracted from A549 cells 5 days after infection with the lentivirus constructs using TRIzol ${ }^{\circledR}$ reagent (Invitrogen Life Technologies). cDNA was synthesized from total RNA using M-MLV reverse transcriptase with random primers according to the manufacturer's instructions (Promega). qPCR analysis was performed using a SYBR Green Master mix kit (Applied Biosystems, Foster City, CA, USA) on a Bio-Rad connect Real-Time PCR platform (Bio-Rad Laboratories, Inc., Hercules, CA, USA). In brief, each PCR reaction mixture, containing $10 \mu \mathrm{l}$ of $2 \mathrm{X}$ SYBR Green Master mix, $1 \mu 1$ of sense and antisense primers $(5 \mu \mathrm{mol} / \mu \mathrm{l})$ and $1 \mu \mathrm{l}$ of cDNA (10 ng), was run for 40 cycles with denaturation at $95^{\circ} \mathrm{C}$ for $15 \mathrm{sec}$, annealing at $58^{\circ} \mathrm{C}$ for $10 \mathrm{sec}$ and extension at $72^{\circ} \mathrm{C}$ for $30 \mathrm{sec}$ in a total volume of $20 \mu$ l. For relative quantification, $2^{-\Delta \Delta \mathrm{CT}}$ was calculated and used as an indication of the relative expression levels, which was calculated by subtracting the $\mathrm{CT}$ values of the control gene from the $\mathrm{CT}$ values of ADAM9. The primer sequences for the PCR amplification of the ADAM9 gene were: ADAM9, sense 5'-TGTGGGAACAGTGTGTTCAAGGA-3' and antisense 5 '-CCAATTCATGAGCAACAATGGAAG-3'. $\beta$-actin was used as an internal control. The primer sequences for $\beta$-actin were: $\beta$-actin, sense 5'-GTGGACATCCGCAAAGAC-3' and antisense 5'-AAAGGGTGTAACGCAACTA-3'.

Western blot analysis. A549 cells were collected 5 days after infection with the lentivirus constructs and were then washed twice with phosphate-buffered saline (PBS; Sigma-Aldrich) and lysed in RIPA lysis buffer (Sigma-Aldrich). Protein concentrations were determined using Protein Assay reagent (Bio-Rad Laboratories, Inc.). Protein samples (30 mg) were loaded on $8 \%$ sodium dodecyl sulfate-polyacrylamide gels. Following electrophoresis, the proteins were transferred onto polyvinylidene difluoride membranes (Bio-Rad Laboratories, Inc.). Blocking was performed using $5 \%$ non-fat milk in a $1 \mathrm{X}$ mixture of Tris-buffered saline and Tween 20 (Sigma-Aldrich). The membranes were then incubated overnight at $4^{\circ} \mathrm{C}$ with the primary antibodies. Following washing and incubation with sheep anti-mouse horseradish peroxidase-conjugated polyclonal IgG secondary antibody (1:10,000; cat no. RPN4201; Amersham Biosciences, Uppsala, Sweden) for $2 \mathrm{~h}$ at room temperature, blotted proteins were detected using an enhanced chemiluminescence system (Millipore Corporation, Billerica, MA, USA) with the BioSpectrum Imaging System (UVP, Upland, CA, USA).

Cell proliferation assay. To measure the effect of downregulation of ADAM9 by RNAi on cell proliferation, a cell counting kit-8 (CCK-8) assay (Dojindo Laboratories, Kumamoto, Japan) was performed. In brief, A549 cells infected with the ADAM9 shRNA lentivirus (Lv/sh-ADAM9) or the non-silencing shRNA lentivirus (Lv/sh-NC), along with untreated cells, were seeded in 96-well plates at a density of $5 \times 10^{3}$ cells per well. The proliferative activity was determined at the end of different experimental periods $(24,48,72,96$ and $120 \mathrm{~h})$ using the CCK-8 assay according to the manufacturer's instructions. When the media changed from red to yellow, the absorbance value at a wavelength of $450 \mathrm{~nm}$ was detected using an enzyme-linked immunosorbent assay reader (Multiskan EX; Thermo Labsystems, Vantaa, Finland). The experiment was performed at least three times with similar results.

The proliferation rate of cells was determined by measuring the incorporation of 5-bromodeoxyuridine (BrdU) into the genomic DNA. In brief, A549 cells infected with Lv/sh-ADAM9 or Lv/sh-NC, along with untreated cells, were 
seeded in a 96 -well plates at a density of $2 \times 10^{3}$ cells per well. A BrdU incorporation assay was performed using a BrdU Cell Proliferation Assay kit (Chemicon, Temecula, CA, USA) according to the manufacturer's instructions. Briefly, $20 \mu \mathrm{l}$ of $1 / 500$ diluted BrdU was added and the assay was incubated for $6 \mathrm{~h}$. Subsequently, $100 \mu \mathrm{l}$ of $1 / 200$ diluted anti-BrdU provided by the BrdU Cell Proliferation Assay kit (Chemicon) and peroxidase-conjugated goat anti-mouse polyclonal IgG antibodies (1:1,000 dilution; cat. no. Ig-0296G; Sigma-Aldrich) were used according to the manufacturer's instructions. The plates were washed twice with PBS containing $0.1 \%$ Triton X-100 (Sigma-Aldrich) and then $100 \mu 1$ 3,3',5,5'-tetramethylbenzidine peroxidase substrate (Sigma-Aldrich) was added. Plates were read at a dual wavelength of $450 / 550 \mathrm{~nm}$ and the growth rate of cells was calculated as described previously (25).

Apoptosis analysis. To measure the effect of lentivirus-mediated siRNA targeting ADAM9 on the cell apoptosis of A549 cells, a terminal deoxynucleotidyl transferase dUTP nick end labeling (TUNEL) assay was performed. In brief, cellular DNA fragmentation was measured with the ApoTag Red in situ Apoptosis detection kit (Chemicon International, Billerica, MA, USA) according to the manufacturer's instructions when A549 cells were infected with Lv/sh-ADAM9 or Lv/sh-NC for $48 \mathrm{~h}$. To quantify the apoptotic cells, the TUNEL-positive cells were counted using a confocal microscope (BX53; Olympus).

In addition, at the molecular level, caspase-3, caspase- 8 and caspase- 9 activity was subsequently detected by ELISA as an additional indicator of apoptosis.

Caspase activity. The activity of caspase- 3 , caspase- 8 and caspase-9 was determined using caspase colorimetric protease assay kits (Millipore Corporation) according to the manufacturer's instructions. In brief, A549 cells were infected with Lv/sh-ADAM9 or Lv/sh-NC for $24 \mathrm{~h}$. Following treatment, cells were washed twice with ice-cold PBS and harvested by centrifugation at $1,000 \mathrm{x}$ g for $5 \mathrm{~min}$. The cell pellets were then lysed in $150 \mu \mathrm{l}$ buffer provided in the kit. Protein concentrations of lysates were measured by the Lowry method. An aliquot of lysates $(80 \mu \mathrm{l})$ was incubated with $10 \mu \mathrm{l}$ substrate of each caspase at $37^{\circ} \mathrm{C}$ for $2 \mathrm{~h}$. Samples were analyzed at $405 \mathrm{~nm}$ in a microplate reader (Thermo Fisher Scientific Inc., Waltham, MA, USA). The relative caspase activity of the control group was referred to as 100 .

Wound healing assay. To assess the effect of downregulation of ADAM9 on cell migration, a wound healing assay was performed. In brief, A549 cells were infected with Lv/sh-ADAM9 or Lv/sh-NC, along with untreated cells, seeded in a $6 \mathrm{~cm}$ dish with $1.5 \times 10^{6}$ wells per dish and cultured for $24 \mathrm{~h}$. A linear wound of the cellular monolayer was created by scratching the confluent cell monolayer using a plastic pipette tip. The monolayer of scratched cells was washed with PBS to remove debris. Following incubation at $37^{\circ} \mathrm{C}$ with $5 \% \mathrm{CO}_{2}$ for $24 \mathrm{~h}$, images of the area of migration were captured under a light microscope (CX41; Olympus) for evaluation. All experiments were performed in triplicate.

Transwell migration assay. The migration assay of A549 cells was performed in vitro using Transwell chambers (Corning
Life Sciences, Tewksbury, MA, USA) in which the two chambers were separated by a Matrigel-coated polycarbonate membrane ( $8 \mu \mathrm{m}$ pore size). In brief, A549 cells infected with Lv/sh-ADAM9 or Lv/sh-NC, along with untreated cells, were seeded into the cell culture insert $(8 \mu \mathrm{m}$ pore size; Falcon, BD Biosciences, Franklin Lakes, NJ, USA), precoated with $25 \mu \mathrm{l}$ of $20 \%$ Matrigel (2-3 $\mathrm{mg} / \mathrm{ml}$ protein) and then placed in a $24-w e l l$ plate (Falcon) with $1 \times 10^{5}$ wells per well. Following culturing cells at $37^{\circ} \mathrm{C}$ for $40 \mathrm{~h}$, they were fixed and stained with $0.5 \%$ crystal violet. The cells on the top of the cell culture insert were removed by wiping with a cotton swab and cell invasion was observed using an immunofluorescence microscope (CKX41; Olympus) by counting the cells that had invaded into the bottom of the cell culture insert. All experiments were performed in triplicate.

Tumor xenograftassay. All animal experiments were performed following the standards of animal care as outlined in the Guide for the Care and Use of Experimental Animals of Jilin University (Changchun, China), following a protocol approved by the Ethics Committees of the Disease Model Research Center, The First Hospital of Jilin University. Approximately 6 week-old male BALB/c nude mice were maintained under specific pathogen-free conditions and provided with food and water ad libitum.

In vitro cultured A549 cells were harvested and a tumorigenic dose of $2 \times 10^{6}$ cells was injected intraperitoneally into $\mathrm{BALB} / \mathrm{c}$ mice. Tumor volume was calculated using the following formula: Tumor volume $=$ length $\mathrm{x}$ width ${ }^{2} / 2$. When tumors grew to an average volume of $75 \mathrm{~mm}^{3}$, mice were randomly divided into the Lv/sh-ADAM9 group, control group (untreated group) and the Lv/sh-NC group ( $\mathrm{n}=10$ in each group). The Lv/ sh-ADAM9 group and Lv/sh-NC group were administered with Lv/sh-ADAM9 or Lv/sh-NC plus PBS in a total volume of $20 \mu \mathrm{l}$ (10 $\mu \mathrm{l}$ virus plus $10 \mu \mathrm{l} \mathrm{PBS}$ ) once a week for 3 weeks, respectively. The control group were administered $20 \mu \mathrm{l}$ PBS once a week for 3 weeks. Following sacrificing the mice, the tumor weight was measured 21 days after treatment. Tumor volume was measured prior to administration of the treatment injections and on days 7,14 and 21 of treatment. The primary tumors were measured and western blot analysis was performed for ADAM9 protein expression. Subsequently, TUNEL staining was performed on $5 \mu \mathrm{m}$ sections of the excised tumors using an ApoTag Red in situ Apoptosis detection kit (Chemicon International) according to the manufacturer's instructions.

Statistical analysis. All data are expressed as the mean \pm standard deviation. Statistical analysis between two samples was performed using Student's t-test. Statistical comparison of more than two groups was performed using one-way analysis of variance followed by Tukey's post hoc test. Graphpad Prism 6.0 software (GraphPad Software, San Diego, CA, USA) and SPSS $^{\circledR} 19.0$ (SPSS, Inc., Chicago, IL, USA) for Windows ${ }^{\circledR}$ were used for statistical analyses. $\mathrm{P}<0.05$ was considered to indicate a statistically significant difference.

\section{Results}

ADAM9 silencing inhibits ADAM9 expression in A549 cells. The lentivirus carrying the ADAM9 siRNA or negative 
A

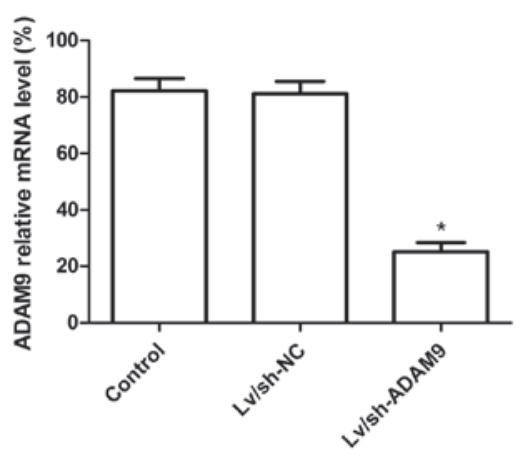

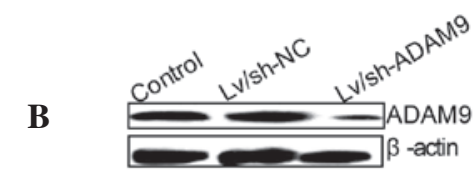

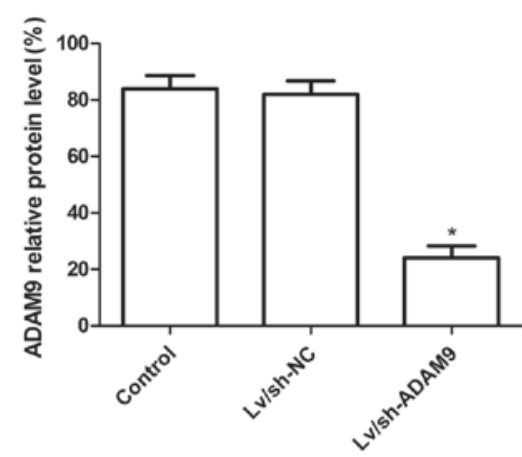

Figure 1. Detection of ADAM9 mRNA and protein expression by RT-qPCR and western blot analysis, respectively. (A) RT-qPCR analysis of ADAM9 mRNA expression in A549 cells following infection with Lv/sh-ADAM9 or Lv/sh-NC. (B) Western blot analysis of ADAM9 protein expression in A549 cells following infection with Lv/sh-ADAM9 or Lv/sh-NC ( $\beta$-actin was included as an internal control). ${ }^{*} \mathrm{P}<0.05$, versus the control group. RT-qPCR, reverse transcription quantitative polymerase chain reaction; ADAM9, A disintegrin and metalloproteinase 9; Lv/sh-ADAM9, ADAM9 shRNA lentivirus; Lv/sh-NC, non-silencing shRNA lentivirus.

control siRNA was infected into A549 cells for 5 days and then $A D A M 9$ expression at the mRNA and protein levels was determined by RT-qPCR and western blot analysis, respectively. RT-qPCR analysis demonstrated that the expression level of ADAM9 mRNA in Lv/sh-ADAM9-infected A549 cells was decreased compared with that in untreated cells or Ad/sh-NC-infected cells ( $\mathrm{P}<0.05$; Fig. 1A). At the protein level, similar results were obtained by western blotting using anti-ADAM9 antibody $(\mathrm{P}<0.05$; Fig. 1B). These data suggested that lentivirus-mediated siRNA targeting ADAM9 could specifically and significantly inhibit the gene expression of ADAM9 in A549 cells.

ADAM9 silencing inhibits cell proliferation in A549 cells. To further assess the role of ADAM9 in regulating A549 cell proliferation, CCK-8 assays were performed on A549 cells following adenovirus infection for 5 days. Fig. 2A shows that no statistically significant differences were observed in cell viability between uninfected cells and cells infected with Lv/sh-NC, indicating that the adenoviral system itself had no cytotoxic effect on cells, while the viability of A549 cells was markedly inhibited by ADAM9 silencing $(\mathrm{P}<0.05$, compared with the control). The inhibitory effect of Lv/sh-ADAM9 on cell proliferation can be observed beginning on day 2 and became more prominent on days 4 and 5 ( $\mathrm{P}<0.05$; Fig. 2A). In addition, BrdU incorporation assays also demonstrated that the inhibition of ADAM9 expression significantly reduced the growth rate of A549 cells during the $48 \mathrm{~h}$ incubation period ( $\mathrm{P}<0.05$; Fig. $2 \mathrm{~B}$ ). These findings suggest that ADAM9 silencing markedly decreased the proliferative ability of A549 cells.

ADAM9 silencing induces cell apoptosis in A549 cells. To investigate the effect of ADAM9 silencing on cell apoptosis in A549 cells, a TUNEL assay was performed. Compared with the control group and Lv/sh-NC group, cell apoptosis was significantly increased in the Lv/sh-ADAM9 group $(\mathrm{P}<0.05$;
Fig. 3A). Subsequently, the effects of ADAM9 silencing on caspase-3, caspase- 8 and caspase- 9 activity were analyzed in A549 cells. As shown in Fig. 3B-D, caspase-3, caspase-8 and caspase-9 activity in the Lv/sh-ADAM9 treatment group significantly increased compared with that in the control group or Lv/sh-NC group $(\mathrm{P}<0.05)$. These results suggest that ADAM9 silencing can induce cell apoptosis in A549 cells.

ADAM9 silencing inhibits cell migration and invasion in A549 cells. To ascertain the inhibitory effect of ADAM9 silencing on A549 cell motility in vitro, a wound-healing assay was performed to investigate the effects on the migration potential of A549 cells. A scratch was introduced into confluent monolayers of different treatment groups and the time-dependent movement of cells into the injured area was monitored microscopically. Cells in the control and Lv/sh-NC groups began migrating $8 \mathrm{~h}$ after scratching. After $24 \mathrm{~h}$, cells in the Lv/sh-ADAM9 group migrated significantly less than those of the control and Lv/sh-NC groups $(\mathrm{P}<0.05$; Fig. $4 \mathrm{~A}$ and $\mathrm{B})$.

The ability of ADAM9 silencing to reduce the invasiveness of A549 cells was further investigated by the transwell system assay. The results demonstrated that invasion was also decreased significantly in the Lv/sh-ADAM9 treatment group compared with the control and the Lv/sh-NC groups $(\mathrm{P}<0.05$; Fig. 4C and D).

ADAM9 silencing suppresses tumor growth in vivo. The in vivo therapeutic efficacy of downregulation of ADAM9 was assessed in male BALB/c mice bearing A549 tumor cells. Tumor growth was monitored for 21 days. At 1 day after the end of treatment, animals were sacrificed and final tumor weights and tumor volume were determined. It was found that the tumor weight of the Lv/sh-ADAM9 group was significantly lower than those of the control group and Lv/sh-NC group (Fig. 5A). In addition, the present study also found that tumor volume following infection with Lv/sh-ADAM9 
A

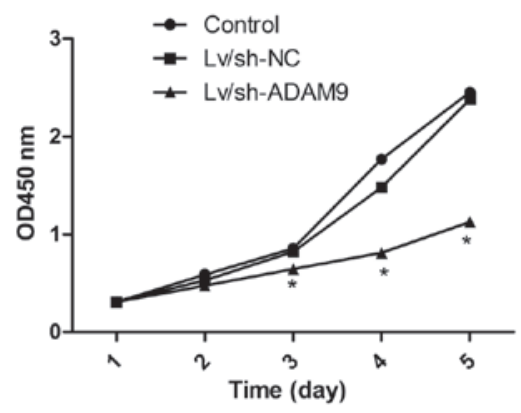

B

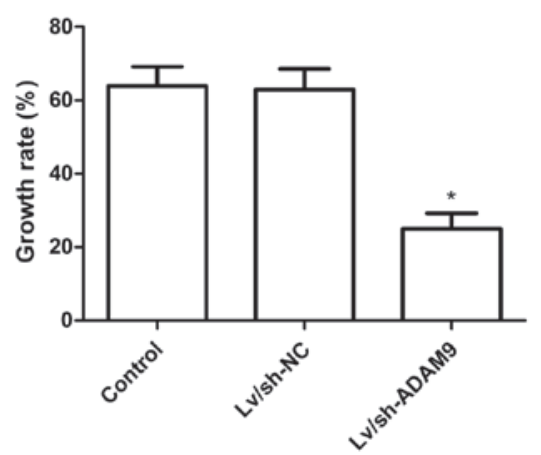

Figure 2. ADAM9 silencing inhibits A549 cell proliferation. (A) ADAM9 silencing resulted in growth inhibition as detected using a cell counting kit-8 assay in A549 cells. (B) ADAM9 silencing led to A549 cell growth inhibition as detected using a 5-bromodeoxyuridine incorporation assay. The growth rate of A549 cells was calculated. All assays were performed in triplicate. ${ }^{*} \mathrm{P}<0.05$, versus the control. ADAM9, A disintegrin and metalloproteinase 9 ; OD, optical density; Lv/sh-ADAM9, ADAM9 shRNA lentivirus; Lv/sh-NC, non-silencing shRNA lentivirus.

A

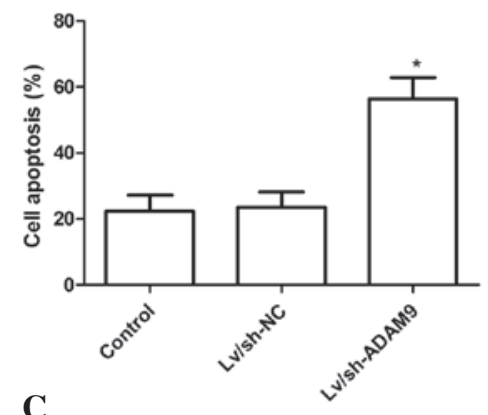

C

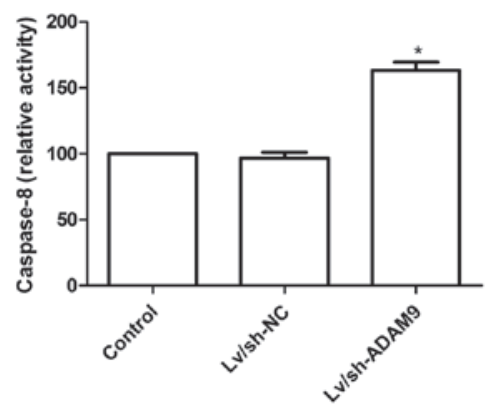

B

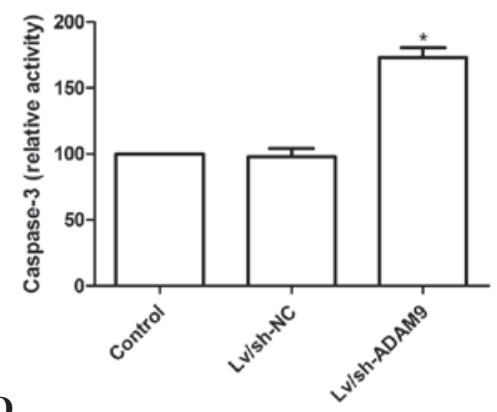

D

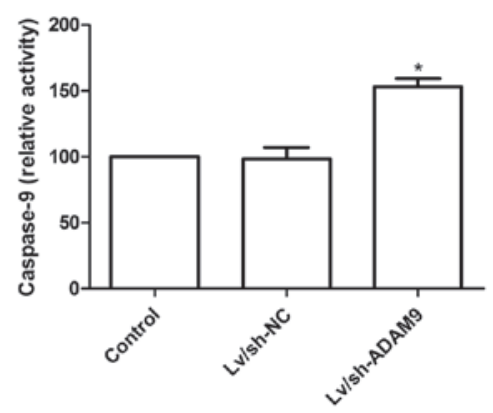

Figure 3. ADAM9 silencing induces cell apoptosis in A549 cells. (A) Terminal deoxynucleotidyl transferase dUTP nick end labeling analysis of A549 cell apoptosis $48 \mathrm{~h}$ after infection with Lv/sh-ADAM9 or Lv/sh-NC. ELISA analysis of (B) caspase-3, (C) caspase-8 and (D) caspase-9 activity following infection with Lv/sh-ADAM9 or Lv/sh-NC. *P<0.05, versus the control. ADAM9, A disintegrin and metalloproteinase 9; Lv/sh-ADAM9, ADAM9 shRNA lentivirus; Lv/sh-NC, non-silencing shRNA lentivirus.

was significantly lower compared with the control group and Lv/sh-NC group ( $\mathrm{P}<0.05$; Fig. 5B).

In addition, in the present study, the expression of ADAM9 in grafted tumor tissues was also examined by western blot analysis. It was found that ADAM9 protein expression levels were decreased in the Lv/sh-ADAM9 treatment group compared with the control group and Lv/sh-NC group (Fig. 5C).

The ADAM9 silencing effects on tumor tissue cell apoptosis were also determined in vivo by TUNEL. The results demonstrated that ADAM9 silencing could significantly induce cell apoptosis compared with the control group and Lv/sh-NC group $(\mathrm{P}<0.05$; Fig. 5D). These results indicate that ADAM9 silencing could suppress the tumor growth of NSCLC in a mouse model.

\section{Discussion}

ADAMs are membrane-anchored enzymes with critical roles in the proteolytic processing of numerous membrane-bound molecules that include growth factors, receptors and adhesion molecules, which affect multiple cellular processes, including cell proliferation, differentiation, migration and invasion (26). Due to their ability to rapidly affect signaling activities between cells and their environment, ADAMs have been involved in a range of human diseases, including cancer $(27,28)$ 
A

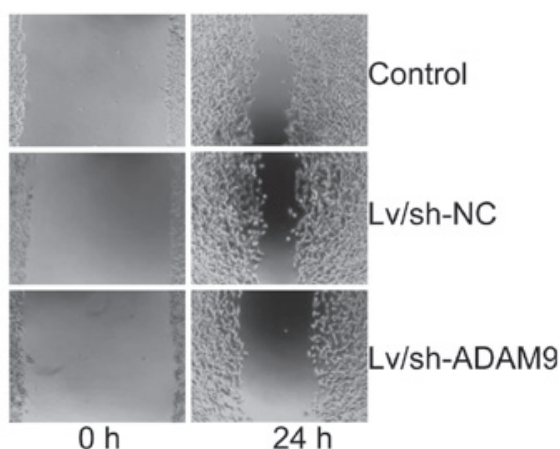

C

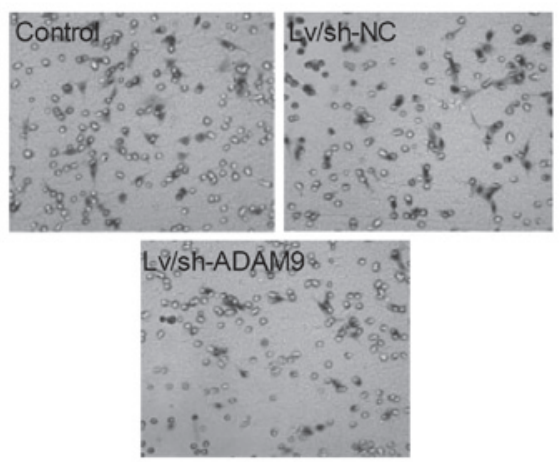

B

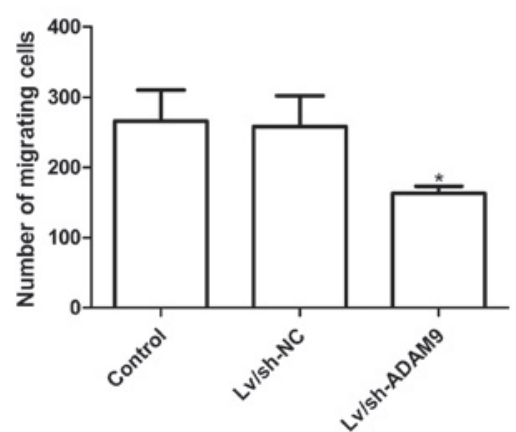

D

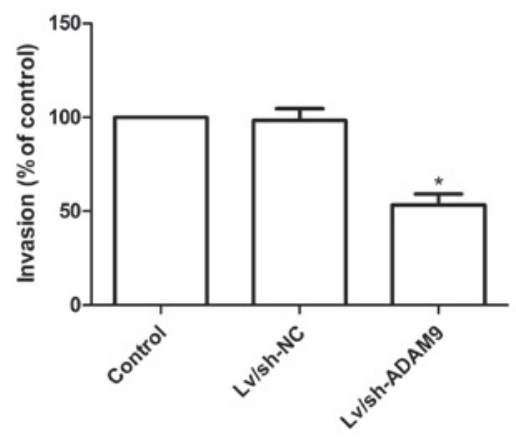

Figure 4. ADAM9 silencing inhibits cell migration and invasion. (A) Cell migration was determined by a wound-healing assay following infection with Lv/sh-ADAM9 or Lv/sh-NC. Phase micrographs of cells were captured at 0 and $24 \mathrm{~h}$ after monolayer wounding (magnification, x20). (B) The number of migrating cells was counted. (C) Cell invasion was determined by Matrigel following infection with Lv/sh-ADAM9 or Lv/sh-NC (magnification, x200). (D) Histogram of the percentage of invaded cells in each group, normalized to the control following infection with Lv/sh-ADAM9 or Lv/sh-NC. "P<0.05, versus the control. ADAM9, A disintegrin and metalloproteinase 9; Lv/sh-ADAM9, ADAM9 shRNA lentivirus; Lv/sh-NC, non-silencing shRNA lentivirus.

A
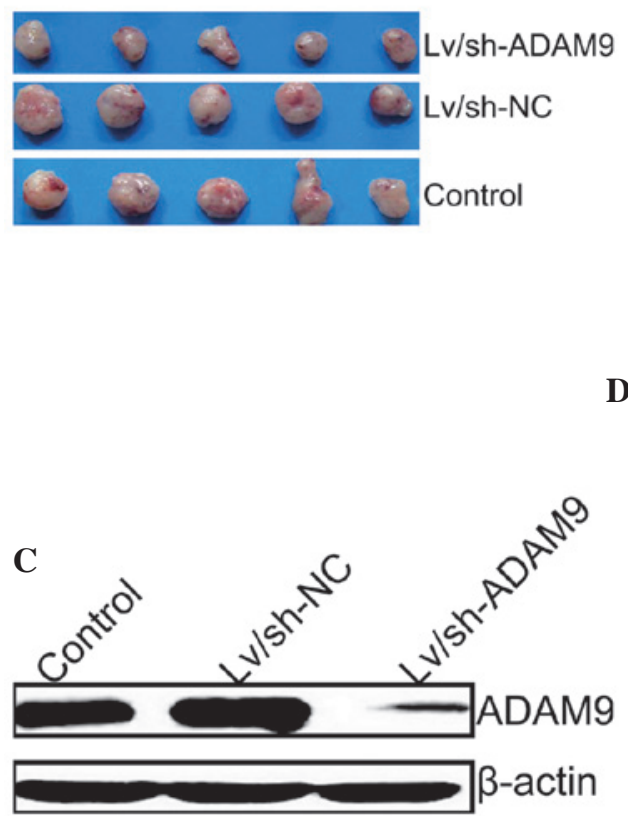

B

D
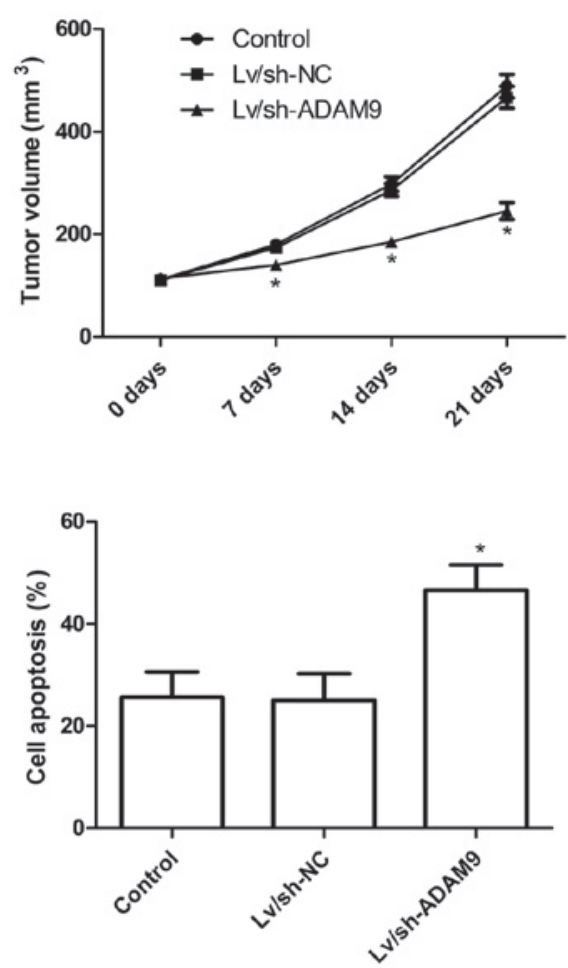

Figure 5. ADAM9 silencing inhibits lung tumor growth in a nude mouse model. (A) Tumor images of mice 21 days following treatment with Lv/sh-ADAM9 or Lv/sh-NC. (B) Tumor volume of mice 7, 14 and 21 days after treatment with Lv/sh-ADAM9 or Lv/sh-NC. (C) Western blot analysis for ADAM9 expression levels in tumor tissue. $\beta$-actin was included as a loading control. (D) Terminal deoxynucleotidyl transferase dUTP nick end labeling assay of cell apoptosis in vivo. ${ }^{*} \mathrm{P}<0.05$, versus the control. ADAM9, A disintegrin and metalloproteinase 9; Lv/sh-ADAM9, ADAM9 shRNA lentivirus; Lv/sh-NC, non-silencing shRNA lentivirus. 
and represent attractive therapeutic targets for the treatment of various types of cancer. ADAM9 an important member of the ADAM family, is frequently upregulated in various types of cancer, including NSCLC and is involved in cancer progression and metastasis (14-21). In line with this opinion, our results demonstrated that downregulation of ADAM9 by RNAi inhibited A549 cell proliferation, migration and invasion in vitro. The results further demonstrated that ADAM9 is important in tumor progression.

Although ADAM9 has not been identified as the principal sheddase of any given substrate so far, it is an active proteinase capable of shedding various molecules with important roles in cancer development and angiogenesis. For example, Peduto et al reported that ADAM9 promotes the proliferation of prostate cancer cells through hydrolyzing and releasing ligands for the epidermal growth factor receptor and fibroblast growth factor receptor on the surface of exfoliative cells (29). Furthermore, Shintani et al suggested that ADAM9 may enhance the adhesion and invasive abilities of tumor cells through its regulation of cell adhesion molecules and altering the sensitivity of tumor cells to growth factors, thereby promoting tumor cell metastasis to the brain (22). A previous study demonstrated that downregulation of ADAM9 by RNAi in prostate cancer cells results in the impairment of proliferation and the osteolytic reaction, as well as causing G1/G0 cell cycle arrest. The G1-to-S phase negative regulators $\mathrm{p} 21^{\mathrm{Cip} 1 / \mathrm{WAF} 1}$ and $\mathrm{p} 27^{\mathrm{Kip} 1}$ acted as downstream targets of the ADAM9-REG4 signaling pathway (30). Hamada et al demonstrated that re-expression of miR-126 and siRNA-based knockdown of ADAM9 in pancreatic cancer cells resulted in reduced cellular migration, invasion and induction of an epithelial marker, and that the miR-126/ADAM9 axis is important in the inhibition of invasive growth of pancreatic cancer cells (31). The present study demonstrated that ADAM9 silencing significantly inhibited the migration and invasion capacity of A549 human lung cancer cells. These studies, in combination with the results reported in the present study, support the possibility that downregulating ADAM9 may contribute to the inhibition of cancer cell invasion and metastasis.

Previously, the successful use of siRNA in downregulating gene expression in several model systems has led to numerous attempts to examine this methodology in a potentially therapeutic setting and this method has been widely used to investigate gene function for cancer therapy $(32,33)$. To examine the possibility of ADAM9 as an effective therapeutic target, endogenous ADAM9 expression was silenced using lentivirus-mediated siRNA targeting the ADAM9 gene in A549 cells and analyzed its anti-cancer effect. Our results demonstrated that downregulation of ADAM9 expression by RNAi inhibits proliferation, migration and invasion, induces apoptosis and decreases tumor growth in a nude mouse model.

In conclusion, in the present study, the results demonstrated that downregulation of ADAM9 expression using lentivirus-mediated siRNA targeting ADAM9 significantly inhibited cell proliferation, migration and cell invasion, induced cell apoptosis in vitro and suppressed tumor growth in a nude mouse model. Considering the significance of cell invasion in metastatic progression, ADAM9 is thus a potential therapeutic target for the treatment of NSCLC.

\section{Acknowledgements}

This study was supported by the Science and Technology Research and innovation team funded by Jilin province (grant no. JL2013528).

\section{References}

1. Jemal A, Bray F, Center MM, Ferlay J, Ward E and Forman D: Global cancer statistics. CA Cancer J Clin 61: 69-90, 2011.

2. Jemal A, Siegel R, Xu J and Ward E: Cancer statistics, 2010. CA Cancer J Clin 60: 277-300, 2010.

3. Reungwetwattana T, Weroha SJ and Molina JR: Oncogenic pathways, molecularly targeted therapies and highlighted clinical trials in non-small-cell lung cancer (NSCLC). Clin Lung Cancer 13: 252-266, 2012.

4. Siegel R, Naishadham D and Jemal A: Cancer statistics, 2013. CA Cancer J Clin 63: 11-30, 2013.

5. Hayama M, Chida M, Karube Y, et al: One-step nucleic acid amplification for detection of lymph node metastasis in lung cancer. Ann Thorac Cardiovasc Surg 20: 181-184, 2014.

6. Coleman RE: Clinical features of metastatic bone disease and risk of skeletal morbidity. Clin Cancer Res 12: 6243-6249, 2006.

7. Sun DS, Hu LK, Cai Y, et al: A systematic review of risk factors for brain metastases and value of prophylactic cranial irradiation in non-small cell lung cancer. Asian Pac J Cancer Prev 15: 1233-1239, 2014.

8. Seals DF and Courtneidge SA: The ADAMs family of metalloproteases: multidomain proteins with multiple functions. Genes Dev 17: 7-30, 2003.

9. Roy M, Luo YH, Ye M and Liu J: Nonsmall cell lung cancer therapy: insight into multitargeted small-molecule growth factor receptor inhibitors. Biomed Res Int 2013: 964743, 2013.

10. Ambrosini G, Adida C and Altieri DC: A novel anti-apoptosis gene, survivin, expressed in cancer and lymphoma. Nat Med 3: 917-921, 1997.

11. Becherer JD and Blobel CP: Biochemical properties and functions of membrane-anchored metalloprotease-disintegrin proteins (ADAMs). Curr Top Dev Biol 54: 101-123, 2003.

12. Duffy MJ, McKiernan E, O'Donovan N and McGowan PM: Role of ADAMs in cancer formation and progression. Clin Cancer Res 15: 1140-1144, 2009.

13. Peduto L: ADAM9 as a potential target molecule in cancer. Curr Pharm Des 15: 2282-2287, 2009.

14. O'Shea C, McKie N, Buggy Y, Duggan C, Hill AD, McDermott E, O'Higgins N and Duffy MJ: Expression of ADAM-9 mRNA and protein in human breast cancer. Int J Cancer 105: 754-761, 2003.

15. Tannapfel A, Anhalt K, Häusermann P, Sommerer F, Benicke M, Uhlmann D, Witzigmann H, Hauss J and Wittekind C: Identification of novel proteins associated with hepatocellular carcinomas using protein microarrays. J Pathol 201: 238-249, 2003.

16. Carl-McGrath S, Lendeckel U, Ebert M, Roessner A and Röcken C: The disintegrin-metalloproteinases ADAM9, ADAM12 and ADAM15 are upregulated in gastric cancer. Int J Oncol 26: 17-24, 2005.

17. Grützmann R, Lüttges J, Sipos B, Ammerpohl O, Dobrowolski F, Alldinger I, Kersting S, Ockert D, Koch R, Kalthoff H, Schackert HK, Saeger HD, et al: ADAM9 expression in pancreatic cancer is associated with tumour type and is a prognostic factor in ductal adenocarcinoma. Br J Cancer 90: 1053-1058, 2004.

18. Fritzsche FR, Jung M, Tölle A, Wild P, Hartmann A, Wassermann K, Rabien A, Lein M, Dietel M, Pilarsky C, Calvano D, Grützmann R, et al: ADAM9 expression is a significant and independent prognostic marker of PSA relapse in prostate cancer. Eur Urol 54: 1097-1106, 2008.

19. Fritzsche FR, Wassermann K, Jung M, Tölle A, Kristiansen I, Lein M, Johannsen M, Dietel M, Jung K and Kristiansen G: ADAM9 is highly expressed in renal cell cancer and is associated with tumour progression. BMC Cancer 8: 179, 2008.

20. Zubel A, Flechtenmacher C, Edler L and Alonso A: Expression of ADAM9 in CIN3 lesions and squamous cell carcinomas of the cervix. Gynecol Oncol 114: 332-336, 2009.

21. Zhang J, Qi J, Chen N, Fu W, Zhou B and He A: High expression of a disintegrin and metalloproteinase-9 predicts a shortened survival time in completely resected stage I non-small cell lung cancer. Oncol Lett 5: 1461-1466, 2013. 
22. Shintani Y,Higashiyama S, Ohta M,Hirabayashi H, Yamamoto S, Yoshimasu T, Matsuda H and Matsuura N: Overexpression of ADAM9 in non-small cell lung cancer correlates with brain metastasis. Cancer Res 64: 4190-4196, 2004.

23. Xu Q, Liu X, Cai Y, Yu Y and Chen W: RNAi-mediated ADAM9 gene silencing inhibits metastasis of adenoid cystic carcinoma cells. Tumour Biol 31: 217-224, 2010.

24. Micocci KC, Martin AC, Montenegro Cde F, et al: ADAM9 silencing inhibits breast tumor cell invasion in vitro. Biochimie 95: 1371-1378, 2013.

25. Lin Y, Peng S and Yu H: RNAi-mediated downregulation of NOB1 suppresses the growth and colony-formation ability of human ovarian cancer cells. Med Oncol 29: 311-317, 2012.

26. Klein $\mathrm{T}$ and Bischoff R: Active metalloproteases of the A Disintegrin and Metalloprotease (ADAM) family: biological function and structure. J Proteome Res 10: 17-33, 2011.

27. Murphy G: The ADAMs: signalling scissors in the tumour microenvironment. Nat Rev Cancer 8: 929-941, 2008.
28. Rocks N,Paulissen G,El Hour M, et al: Emerging roles of ADAM and ADAMTS metalloproteinases in cancer. Biochimie 90: 369-379, 2008

29. Peduto L, Reuter VE, Shaffer DR, Scher HI and Blobel CP: Critical function for ADAM9 in mouse prostate cancer. Cancer Res 65: 9312-9319, 2005.

30. Liu CM, Hsieh CL, He YC, et al: In vivo targeting of ADAM9 gene expression using lentivirus-delivered shRNA suppresses prostate cancer growth by regulating REG4 dependent cell cycle progression. PLoS One 8: e53795, 2013.

31. Hamada S, Satoh K, Fujibuchi W, et al: MiR-126 acts as a tumor suppressor in pancreatic cancer cells via the regulation of ADAM9. Mol Cancer Res 10: 3-10, 2012.

32. Zhang J and Hua ZC: Targeted gene silencing by small interfering RNA-based knock-down technology. Curr Pharm Biotechnol 5: 1-7, 2004.

33. Uprichard SL: The therapeutic potential of RNA interference. FEBS Lett 579: 5996-6007, 2005. 